\title{
EL LINFANGIOMA CAVERNOSO DE LOS GENITALES EXTERNOS
}

\author{
J.A. SOTELINO*, E. DE DIOS MONTOTO**, K. WEICHERT-JACOBSEN*** \\ *Servicio de Urología. Christian-Albrechts-Universität zu Kiel. Kiel. Alemania Federal. \\ **Servicio de Obstetricia y Ginecología. Hospital General de Móstoles. Móstoles. Madrid (España). \\ ${ }^{* * *}$ Servicio de Urología. Hospital Herford. Herford. Alemania Federal.
}

Actas Urol Esp. 28 (9): 698-701, 2004

\section{RESUMEN}

EL LINFANGIOMA CAVERNOSO DE LOS GENITALES EXTERNOS

INTRODUCCIÓN: Tumores fluctuantes en la base escrotal y penil pueden ser causados por linfangiomas benignos genitourinarios. Estas malformaciones son muy poco frecuentes y son observadas preferentemente en niños.

MÉTODO Y RESULTADOS: Presentamos un paciente de 10 años de edad que acude a nuestro centro con un tumor azulado, indoloro y fluctuante, con un tamaño de $4 \times 2 \mathrm{~cm}$ en el escroto y la base penil. Por resonancia magnética nuclear fue posible realizar una reconstrucción exacta de la extensión anatómica de esta lesión de imagen hipoecogénica. La extirpación quirúrgica fue realizada sin complicaciones demostrándose en el estudio histológico un linfangioma cavernoso. En la actualidad no se observa recurrencia local (follow up: 6 meses).

CONCLUSIÓN: Un linfangioma cavernoso del escroto o pene es muy poco común. Éste es el segundo caso descrito en la literatura. Incluso linfangiomas de otras características histológicas raramente afectan esta región. Los linfangiomas son tumores benignos de vasos linfáticos. El tejido circundante muestra una reacción inflamatoria con escarificación. En el 50 \% de los casos se considera una lesión congénita. 9/10 linfangiomas se manifiestan antes de los dos años de edad. La terapia es la excisión completa.

PALABRAS CLAVE: Linfangioma cavernoso. Escroto. Diagnóstico. Terapia.

\section{ABSTRACT \\ THE CAVERNOUS LYMPHANGIOMA OF THE EXTERNAL GENITALIA}

PURPOSE: Fluctuating tumours of the scrotal and penile basis can be caused by benign lymphangiomas. These rare malformations can preferently be seen in children.

CASE REPORT: We report about a 10 year old boy, who was presented in our department with a bluish, indolent, $4 \times 2 \mathrm{~cm}$ sized, fluctuating tumor of the scrotum and penile basis. Magnetic resonance imaging could demonstrate the whole anatomic extension of the sonographically hypoechoic lesion. Operative removal of the tumour was performed without complication. Histologically, the specimen was diagnosed as cavernous lymphangioma. To date, there is no sign of recurrence (follow-up period: 6 months).

CONCLUSION: A cavernous lymphangioma of the scrotum or penis is very uncommon. We report, to the best of our knowledge, the 2nd case reported in literature. Even lymphangiomas with other histological features rarely affect this region. Lymphangiomas are benign tumours of lymphatic vessels. The surrounding tissue sometimes shows a perifocal inflammation and scarification. About $50 \%$ of the cases are considered congenital. 9/10 lymphangiomas become obvious during the first 2 postnatal years. Therapy of choice is the complete excision.

KEY WORDS: Cavernous lymphangioma. Scrotum. Diagnosis. Therapy. 
$\mathrm{L}$ os tumores de la base escrotal y pene que aparecen en niños, sugieren como primer diagnóstico, hidroceles, hernias inguinales o varicoceles. Tumores fluctuantes causados por linfangiomas genitourinarios son muy poco frecuentes. Se trata de tumores linfáticos benignos y son observados preferentemente en niños. La diferenciación morfológica únicamente puede realizarse histológicamente. Las características histológicas contienen una estructura linfática multiquística, multilobular o reticulada con anastomosis de finos e irregulares vasos linfáticos, revestidos por endotelio plano. El líquido intraluminar proteínico contiene lípidos con eritrocitos y linfocitos. El tejido conectivo circundante demuestra un conglomerado linfático y una reacción inflamatoria secundaria con tendencia a la fibrosis. En un 50\%-65\% de los casos se trata de una lesión congénita viéndose que un 90\% de los linfangiomas se manifiestan en los dos primeros años de edad ${ }^{2}$. La terapia de esta enfermedad benigna depende de la extensión y de la localización. Únicamente una excisión completa evita el riesgo de recidiva.

\section{CASO CLÍNICo}

Presentamos el caso de un paciente de 10 años que fue diagnosticado a los 3 años de un pequeño tumor fluctuante en la base del pene y del escroto izquierdo, de color azulado que permaneció sin variación de tamaño durante 7 años. El motivo de ingreso en nuestro centro fue el crecimiento rápido y repentino de dicho tumor. El tumor presentaba un tamaño de $4 \times 2 \mathrm{~cm}$, era indoloro, violáceo y se encontraba adherido a la fascia en la base penoescrotal (Fig. 1). Los testículos, la región inguinal al igual que la analítica básica de sangre y orina eran normales.

La ecografía escrotal mostraba un tumor hipoecogénico, homogéneo y perfectamente delimitado. La pielografía, la ecografía renal y del retroperitoneo no mostraron hallazgos patológicos. La imagen en resonancia magnética nuclear (RMN) era la de un tumor multiquístico sin conexión con estructuras vecinas (Fig. 2).

En el acto quirúrgico, macroscópicamente se observaba un tumor poliquístico, subcutáneo, con pequeñas hemorragias, parcialmente relleno de liquido seroso, que se extendía semicircular-

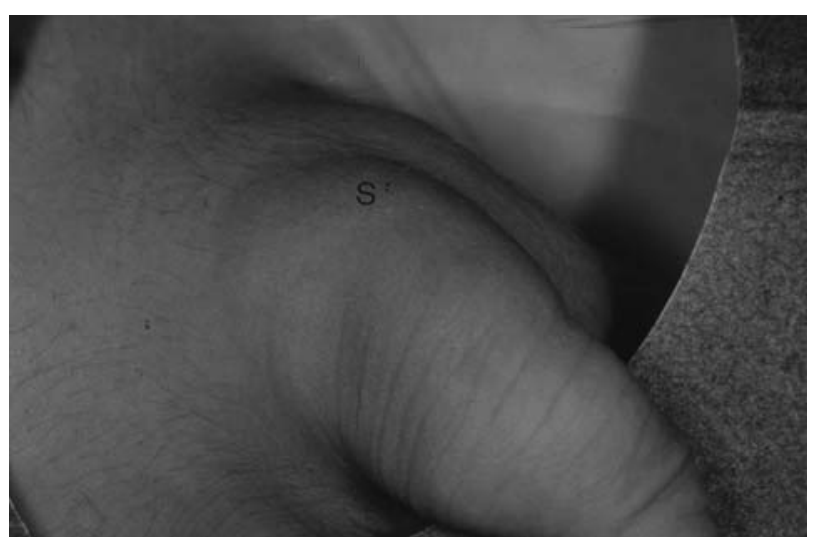

FIGURA 1. Varón de 10 años de edad. Tumor fluctuante, azulado, $e$ inodoloro (S) en el escroto y la base penil.

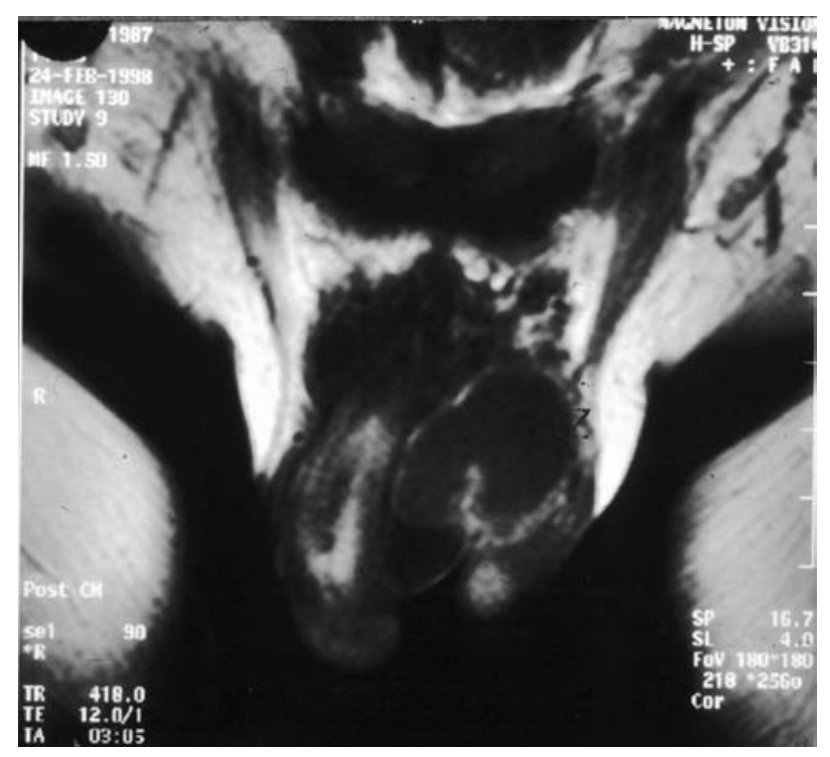

FIGURA 2. Resonancia magnética nuclear (RMN), región genital: tumor poliquístico sin conexión con el cuerpo cavernoso, la uretra o testículos.

mente por la base del pene hasta la sinfisis púbica, llegando dorsalmente hasta la fascia del músculo recto mayor del abdomen. El estudio anatomopatológico del espécimen demostró vasos linfáticos con un lumen ancho con eritrocitos y linfocitos compatible con un linfangioma cavernoso. El post-operatorio transcurrió sin complicaciones. En la actualidad el paciente se encuentra asintomático y no se observa recurrencia local.

\section{DISCUSIÓN}

Los linfangiomas son tumores benignos compuestos de vasos linfáticos con proliferación de endotelio y aglomerados de eritrocitos y linfocitos. 
Cerca de la mitad de estas lesiones son congénitas y en el 90\% de los casos se manifiestan antes de los dos años de edad. La distribución según el sexo es por igual, viéndose en un 95 $\%$ una localización preferente en las regiones cefálica, cervical y axilar. El desarrollo de esta patología en la región urogenital es muy poco frecuente. La sintomatología de la lesión es leve e insidiosa al principio. El síntoma más común es un repentino y rápido crecimiento tumoral $^{1-3}$.

La etiología de estas malformaciones es la proliferación de vasos procedentes de tejidos hamartomatosos aislados e incomunicados. Otras etiologias pueden ser neoplasias o displasias de vasos linfáticos congénitos, así como los debidos a traumatismos o inflamación del drenaje linfáti$\mathrm{co}^{2-5}$.

Los linfangiomas muestran una estructura multiquística, multilocular o esponjosa con anastomosis de finos e irregulares vasos ectásicos. En el tejido que rodea a estos vasos linfáticos, revestidos por un endotelio plano con una musculatura no organizada y con un tejido fibrótico, perifocalmente se observa una reacción inflamatoria y con escarificación ${ }^{6-8,11}$ (Fig. 3).

La clasificación histológica del linfangioma diferencia tres formas ${ }^{1-3,6-8}$ :

1. El linfangioma simple de forma capilar (superficial) con revestimiento de vasos linfáticos finos (región preferente: cabeza, nuca) ${ }^{3,6}$.

2. La forma quística conjunta con una dilatada estructura quística, de tejido colagenoso y con escasas imágenes celulares. Perteneciendo ejem-

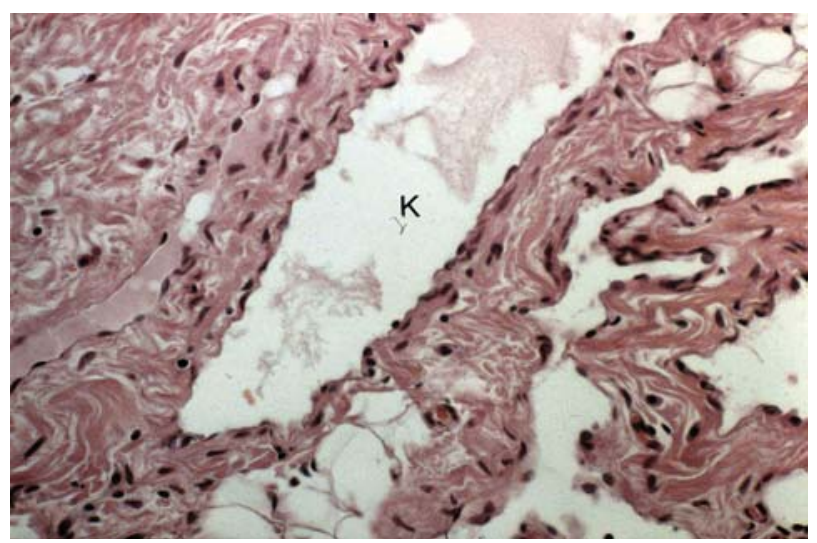

FIGURA 3. Histología de un linfangioma cavernoso (HE, $\mathrm{X}=80$ ): estructura cavernosa (K) con conglomerados de vasos linfáticos dilatados y de crecimiento infiltrativo. plarmente a este tipo histológico el conocido higroma quístico congénito del cuello (región preferente: cuello, pecho y axila).

3. Por último el linfangioma cavernoso con conglomerados de vasos linfáticos dilatados y de crecimiento predominante infiltrativo en dermis y tejido subcutáneo ${ }^{3,6,9}$. Este tipo histológico es el que se presenta en nuestro caso, siendo éste el segundo caso descrito en la literatura ${ }^{5}$.

El diagnóstico se realiza por el examen físico, la ecografía y la tomografía axial computarizada o la resonancia magnética nuclear. En caso de ininteligibles alteraciones en la región del escroto, la resonancia magnética nuclear es el principal aliado diagnóstico, ofreciendo aparte de un exacto diseño anatómico, datos sobre la extensión tumoral y sus relaciones con estructuras vecinas ${ }^{10}$. De interés histórico en el diagnóstico de linfangiomas cutáneos quedan por mencionar las presentaciones gráficas por inyección de partículas de tinta por Tryb (1929), los detallados estudios sobre profundidad y extensión en linfangiomas con ayuda del escintigrama linfático por Penvy (1967) al igual que la linfografía radiológica de la región genitourinaria por Schmid y Greiner $(1971)^{3,11,12}$.

Una regresión espontánea del linfangioma genitourinario no es de esperar. Complicaciones como erisipela, linforrea, etc. son poco frecuentes en esta enfermedad benigna causados en la mayor parte de los casos por lesiones traumáticas ${ }^{11}$. La terapia consiste en una excisión radical ${ }^{2,5,8}$, ya que una excisión incompleta puede ser causa de recidiva $^{2,3}$. La irradiación local al igual que la escleroterapia como terapia inicial son desaconsejables, ya que se acompañan de un muy elevado riesgo de recidiva ${ }^{3,11}$.

\section{REFERENCIAS}

1. Enzinger FM, Weiss SW. Tumors of lymph vessels, en: soft tissue tumors. $3^{\text {rd }}$ Edition; Mosby-Year Book, Inc 1994:679688.

2. Hurwitz RS, Shapiro E, Hulbert WC, Diamond DA, Casale AJ, Rink RC. Scrotal cystic lymphangioma: the misdiagnosed scrotal mass. J Urol 1997;158:1182-1185.

3. Mac Millan RW, Mac Donald BR, Alpern HD. Scrotal lymphangioma. Urology 1984;23:79-80.

4. Merka ST, Bhatt KS, Wood FW. Cystic lymphangioma of the scrotum: a case report. J Urol 1984;131:1179-1181. 
5. Forstner R, Hricak H, Kalbhen CL, Kogan BA, Mcaninch JW. Magnetic resonance imaging of vascular lesions of the scrotum and penis. Urology 1995;46:581-583.

6. Schmid GH, Greiner R. Lymphography of the scrotum and penis in lymphangioma circumscriptum cysticum. Dermatologica 1971;142:184-190.

7. Tryp A. Lymphangiome der Haut. Arch Derm Syph 1929; 158:468-479.

8. Abara EO, Churchill BM, Edwards V, Phillips MJ. Torsion of cavernous lymphangioma: an unusual cause of acute scrotum. J Urol 1989;142:1296-1297.

9. Hajdu SI. Pathology of soft tissue tumors. Lea \& Febiger, Philadelphia, 1979:410-411.

10. Rosai J. En: Ackerman's surgical pathology, $8^{\text {th }}$ Edition; Mosby-Year Book, Inc 1995;2:2070-2073.
11. Aufdermaur M, Baur E, Fassbender H. Pathologie der Gelenke und Weichteiltumoren en: Spezielle pathologische Anatomie, Band 18/II Springer-Verlag, Berlin, Heidelberg, New York, Tokyo, 1984: 1361-1362

12. Calonje E, Wilson-Jones E. Vascular tumors. Tumors and tumor-like conditions of blood vessels and lymphatics en: Lever's Histopathology of the skin $8^{\text {th }}$ Edition; LippinnscottRaven Publishers, Philadelphia 1997: 921-923.

Dr. J.A. Sotelino

Lutterothstr, 3

D-20255 Hamburg (Germany)

(Trabajo recibido el 5 septiembre de 2003) 